\title{
Antibiotic resistance of mixed biofilms in cystic fibrosis: impact of emerging microorganisms on treatment of infection
}

\author{
Susana Patrícia Lopes ${ }^{\mathrm{a}, *}$, Howard Ceri $^{\mathrm{b}}$, Nuno Filipe Azevedo ${ }^{\mathrm{c}}$, Maria Olívia Pereira ${ }^{\mathrm{a}}$ \\ a IBB-Institute for Biotechnology and Bioengineering, Centre for Biological Engineering, Universidade do Minho, Campus de Gualtar 4710-057 Braga, Portugal \\ b Department of Biological Sciences, University of Calgary, 2500 University Dr NW, Calgary, Alberta T2N 1N4, Canada \\ c LEPAE, Department of Chemical Engineering, Faculty of Engineering, University of Porto, Rua Dr Roberto Frias, $4200-465$ Porto, Portugal
}

\section{A R T I C L E I N F O}

\section{Article history:}

Received 10 January 2012

Accepted 30 April 2012

\section{Keywords:}

Cystic fibrosis

Antibiotics

Polymicrobial biofilms

Pseudomonas aeruginosa

\begin{abstract}
A B S T R A C T
Cystic fibrosis (CF) is a genetic disorder associated with multispecies infections where interactions between classical and newly identified bacteria might be crucial to understanding the persistent colonisation in CF lungs. This study investigated the interactions between two emerging species, Inquilinus limosus and Dolosigranulum pigrum, and the conventional CF pathogen Pseudomonas aeruginosa by evaluating the ability to develop biofilms of mixed populations and then studying their susceptibility patterns to eight different antimicrobials. Monospecies biofilms formed by I. limosus and D. pigrum produced significantly less biomass than $P$. aeruginosa and displayed greater sensitivity to antimicrobials. However, when in dual-species biofilms with P. aeruginosa, the emerging species I. limosus and D. pigrum were crucial in increasing tolerance of the overall consortia to most antibiotics, even without a change in the number of biofilm-encased cells. These results may suggest that revising these and other species interactions in $\mathrm{CF}$ might enable the development of more suitable and effective therapies in the future.
\end{abstract}

(C) 2012 Elsevier B.V. and the International Society of Chemotherapy. All rights reserved.

\section{Introduction}

Cystic fibrosis (CF) is a common genetic disease involving the production of thick and sticky mucus that predisposes CF patients to frequent pulmonary infections [1,2]. Pseudomonas aeruginosa is the dominant pathogen colonising older patients in CF [3], often adopting a biofilm mode of growth as a survival strategy. However, recent studies have shown that standard laboratory methods may fail to identify or may misidentify other isolates that are actually present but are usually only detected using molecular biology techniques [4]. Two novel microorganisms that have been increasingly isolated from CF specimens are Inquilinus limosus and Dolosigranulum pigrum. Although available information about these organisms is scarce, their presence is very likely to occur together with conventional pathogens, creating a diverse mosaic of bacteria in the $\mathrm{CF}$ lung [5].

Antibiotic resistance is a well-known phenomenon in chronic infections and is an increasing concern in CF [6]. The intensive selective pressure provided by the large amount of antibiotics to which CF microbial populations are exposed is one factor contributing to such resistance. Typically, the choice of antimicrobials in CF only relies on antibiotic susceptibility testing of the traditional

\footnotetext{
* Corresponding author. Tel.: +351 253601 961; fax: +351253604 429 .

E-mail address: supat@deb.uminho.pt (S.P. Lopes).
}

organism, $P$. aeruginosa. However, the wealth of species of different phenotypes and sensitivities prevailing in the airways of these patients is clearly more complex than a monomicrobial disease and it has been suggested that they can undermine the effectiveness of the treatment commonly practiced, thus resulting in a less than optimum treatment outcome for affected individuals. To the authors' knowledge, this is the first study evaluating the spectrum of antimicrobial resistance of dual-species biofilms involving classical and emerging microorganisms related to CF. The ability of these species to develop single and mixed biofilms was also assessed through biomass and culturable cells analysis and the obtained results were correlated with the susceptibility profiles found for the species involved.

\section{Materials and methods}

\subsection{Bacterial strains, growth media and buffers}

Three CF-related bacterial species were used in this study, the traditional pathogen $P$. aeruginosa PA14 as well as two emerging microorganisms, I. limosus M53 and D. pigrum CIP 104051 (Institute Pasteur Collection, Paris, France). Pseudomonas aeruginosa and I. limosus pure cultures were grown in tryptic soy broth (TSB) (EMD Chemicals Inc., Gibbstown, NJ), whereas brain-heart infusion (BHI) (EMD Chemicals Inc.) was used to culture D. pigrum. Since $P$. aeruginosa and $D$. pigrum require different culture media to grow, 
Table 1

Bacterial cultures and growth conditions used in this study.

\begin{tabular}{|c|c|c|c|c|}
\hline Bacterial culture & Strain & Growth medium & $\begin{array}{l}\text { Incubation } \\
\text { temperature }\left({ }^{\circ} \mathrm{C}\right)\end{array}$ & $\begin{array}{l}\text { Incubation } \\
\text { period (h) }\end{array}$ \\
\hline Pseudomonas aeruginosa & PA14 & TSB/TSA & 37 & 6 \\
\hline Inquilinus limosus & M53 & TSB/TSA & 37 & 48 \\
\hline Dolosigranulum pigrum & CIP 107041 & BHI/TSA & 37 & 72 \\
\hline P. aeruginosa + I. limosus & - & TSB/TSA & 37 & 24 \\
\hline$P$. aeruginos $a+D$. pigrum & - & TSB or BHI/TSA & 37 & 24 \\
\hline
\end{tabular}

TSB, tryptic soy broth; TSA, tryptic soy agar; BHI, brain-heart infusion.

for experiments of mixed biofilms involving these two microorganisms, $P$. aeruginosa was also grown in BHI and D. pigrum was also grown in TSB. The temperature of incubation was $37^{\circ} \mathrm{C}$ and the period of biofilm formation varied for each strain. All conditions used for single and mixed biofilm experiments are summarised in Table 1.

\subsection{In vitro biofilm formation}

Single and mixed biofilms were grown on a Calgary Biofilm Device (CBD) (MBEC Biofilm Technologies, Calgary, Alberta) as previously described by Ceri et al. [7].

\subsection{Analysis of pre-formed biofilms}

Single and mixed biofilms formed on the CBD were further analysed in terms of biomass and number of culturable cells.

\subsubsection{Biomass}

Peg lids were rinsed with double-distilled water and were left to dry for $10 \mathrm{~min}$. Biofilm-growing bacteria on the pegs were then stained with $1 \%(\mathrm{v} / \mathrm{v})$ crystal violet (Sigma-Aldrich, Oakville, ON, Canada) for $1 \mathrm{~min}$ and were washed twice with double-distilled water. Pegs were then decolorised with pure methanol (Sigma) and the optical density at $550 \mathrm{~nm}$ of the obtained solution was measured using a microtitre plate reader (Labequip Ltd., Markham, ON, Canada). At least eight replicates were run for each condition.

\subsubsection{Cell culturability}

The number of adhering bacteria within the biofilm was determined by breaking four pegs of the CBD under aseptic conditions. An Aquasonic Water-table Sonicator (model 250 HT; VWR International, Edmonton, $\mathrm{AB}$, Canada) was used to disrupt the biofilm on the broken pegs submerged in sterile $0.9 \%(\mathrm{v} / \mathrm{v})$ saline complemented with $1 \%(\mathrm{v} / \mathrm{v}$ ) Tween 20 for $10 \mathrm{~min}$ (Sigma). The disrupted biofilms were subsequently serially diluted in saline and plated on tryptic soy agar (TSA) (EMD Chemicals Inc.) for viable cell counting. Selective agar media were used to plate $P$. aeruginosa [Pseudomonas isolation agar (PIA)] and I. limosus [Burkholderia cepacia selective agar (BCSA) supplemented with $300000 \mathrm{IU} / \mathrm{L}$ polymyxin $\mathrm{B}$ and $100 \mathrm{mg} / \mathrm{L}$ ticarcillin]. TSA and PIA plates were incubated for $24 \mathrm{~h}$ (for I. limosus, TSA plates were incubated for $40 \mathrm{~h}$ ), whereas BCSA plates were incubated for $48 \mathrm{~h}$ at $37^{\circ} \mathrm{C}$ before enumeration of colonyforming units (CFU). All samples were run in quadruplicate.

\subsection{Antibiotic stock solutions}

Eight clinically relevant antibiotics were used, including tobramycin, gentamicin, levofloxacin, ciprofloxacin, clindamycin, cefotaxime, chloramphenicol and rifampicin. All antibiotics were from Sigma-Aldrich. Stock solutions of antimicrobial agents were prepared at $5120 \mu \mathrm{g} / \mathrm{mL}$ and then $500 \mu \mathrm{L}$ aliquots were stored at $-70^{\circ} \mathrm{C}$. Working solutions were prepared on the day of use at $1024 \mu \mathrm{g} / \mathrm{mL}$ in cation-adjusted Mueller-Hinton broth
(BD Diagnostics, Franklin Lakes, NJ). For susceptibility testing, antibiotic concentrations ranged from $2 \mu \mathrm{g} / \mathrm{mL}$ to $1024 \mu \mathrm{g} / \mathrm{mL}$.

\subsection{Antibiotic susceptibility testing}

After washing biofilms with saline, a 'challenge plate' was prepared according to Ceri et al. [7]. The minimum inhibitory concentration (MIC) was determined by reading the optical density of the challenge plate at $650 \mathrm{~nm}$, with the exception of those cultures that used sheep blood in the susceptibility testing, where MIC values were determined by visual observation of the turbidity gradient on the challenge plate. This turbidity demonstrates the ability of bacteria to grow as a planktonic population in the presence of antibiotic; hence, the minimum concentration where growth inhibition occurs is equivalent to the MIC value for most organisms $[7,8]$. Minimum biofilm eradication concentration (MBEC) values were determined by enumerating spot plates for bacterial growth.

\subsection{Statistical analysis}

Data were analysed using Prism software v.4.0 for Macintosh (GraphPad Software Inc., La Jolla, CA). The ability of strains to form biofilms was assessed by one-way analysis of variance (ANOVA) tests, and Tukey's post hoc test was performed to subsequently compare pairs of columns. Results were considered statistically significant at $P<0.05$.

\section{Results}

In this study, bacterial biofilms of traditional and emerging CF-related microorganisms were readily formed on the CBD, providing a valuable and reliable technology for selection of clinically effective antibiotics. Susceptibility assays were performed after all biofilms achieved a threshold concentration of $10^{4}-10^{6} \mathrm{CFU} / \mathrm{peg}$, which required different incubation times for each biofilm (Table 1 ).

The concentrations of antibiotic able to inhibit planktonic bacteria (MIC) and those required to kill biofilm-encased bacteria (MBEC) are summarised in Table 2. Most antibiotics were effective in inhibiting planktonic growth of single species at low concentrations; however, mixed planktonic populations required equal or even higher concentrations than those applied to inhibit the planktonic growth of single populations.

Only a few antibiotics were able to kill biofilm bacteria at relatively low concentrations. Generally, MBEC values were significantly greater compared with MIC data, suggesting that once established, biofilms are notoriously difficult to eradicate and high doses of antimicrobials are needed to eliminate them. Monospecies biofilms involving only $P$. aeruginosa were considerably more resistant to most antibiotics tested than those developed by other organisms.

The minimum bactericidal concentration required to kill bacteria in mixed biofilms was generally equal to the concentration needed to kill the more resistant single biofilm of the encompassed species, which was predominately $P$. aeruginosa. Results obtained 
Table 2

In vitro susceptibility patterns of single-species and dual-species cultures of cystic fibrosis-related organisms to eight clinically relevant antibiotics ${ }^{\mathrm{a}}$.

\begin{tabular}{|c|c|c|c|c|c|c|}
\hline Antibiotic & $\begin{array}{l}\text { Pseudomonas } \\
\text { aeruginosa }\end{array}$ & $\begin{array}{l}\text { Inquilinus } \\
\text { limosus }\end{array}$ & $\begin{array}{l}\text { Dolosigranulum } \\
\text { pigrum }\end{array}$ & $\begin{array}{l}\text { P. aeruginosa }+I \text {. } \\
\text { limosus }\end{array}$ & $\begin{array}{l}P . \text { aeruginosa }+D \\
\text { pigrum }(\mathrm{TSB})\end{array}$ & $\begin{array}{l}P . \text { aeruginosa }+D \text {. } \\
\text { pigrum }(\mathrm{BHI})\end{array}$ \\
\hline \multicolumn{7}{|c|}{ Tobramycin } \\
\hline MIC & $<2$ & 128 & 16 & 64 & 16 & 8 \\
\hline MBEC & $>1024$ & 512 & 128 & 256 & $>1024$ & $>1024$ \\
\hline \multicolumn{7}{|c|}{ Gentamicin } \\
\hline MIC & 8 & 16 & 512 & $>1024$ & 16 & 8 \\
\hline MBEC & 128 & 512 & $>1024$ & $>1024$ & $>1024$ & $>1024$ \\
\hline \multicolumn{7}{|c|}{ Levofloxacin } \\
\hline MIC & $<2$ & $<2$ & 4 & $<2$ & 128 & 8 \\
\hline MBEC & $>1024$ & 16 & 256 & $>1024$ & $>1024$ & $>1024$ \\
\hline \multicolumn{7}{|c|}{ Ciprofloxacin } \\
\hline MIC & $<2$ & $<2$ & 256 & $>1024$ & $<2$ & $<2$ \\
\hline MBEC & $>1024$ & 32 & 512 & $>1024$ & 8 & 1024 \\
\hline \multicolumn{7}{|c|}{ Clindamycin } \\
\hline MIC & $>1024$ & 512 & 16 & $>1024$ & $>1024$ & $>1024$ \\
\hline MBEC & $>1024$ & $>1024$ & $>1024$ & $>1024$ & $>1024$ & $>1024$ \\
\hline \multicolumn{7}{|c|}{ Cefotaxime } \\
\hline MIC & 16 & $<2$ & $<2$ & 256 & 32 & 256 \\
\hline MBEC & $>1024$ & $>1024$ & $>1024$ & $>1024$ & $>1024$ & $>1024$ \\
\hline \multicolumn{7}{|c|}{ Chloramphenicol } \\
\hline MIC & 128 & 256 & 4 & $>1024$ & 1024 & 128 \\
\hline MBEC & $>1024$ & 1024 & 32 & $>1024$ & $>1024$ & $>1024$ \\
\hline \multicolumn{7}{|c|}{ Rifampicin } \\
\hline MIC & $>1024$ & $<2$ & $<2$ & $>1024$ & $>1024$ & $>1024$ \\
\hline MBEC & $>1024$ & 8 & 32 & $>1024$ & $>1024$ & $>1024$ \\
\hline
\end{tabular}

MIC, minimum inhibitory concentration; MBEC, minimum biofilm eradication concentration; TSB, tryptic soy broth; BHI, brain-heart infusion.

a MIC and MBEC values expressed in $\mathrm{mg} / \mathrm{L}$.

with selective media enabled the observation that $P$. aeruginosa is the organism prevailing within dual-species biofilms with I. limosus, presenting ca. $70 \%$ of the total cell number. Because there is no information about specific selective media available for $D$. pigrum, no such study was performed for this microorganism. By comparing the susceptibility patterns of $P$. aeruginosa and $D$. pigrum dual-species biofilms growing in different media (Table 2, last two columns), it was observed that antibiotics easily inhibited the growth of planktonic suspensions when TSB was used to culture mixed populations. Susceptibility profiles of these dual-species biofilms in TSB were similar to those obtained with $P$. aeruginosa, showing reduced susceptibility to antibiotics as biofilms. Biofilms growing in BHI did not follow any similar pattern of susceptibility, showing variations in both MIC and MBEC values.

Pseudomonas aeruginosa was clearly the organism that produced the greatest amount of biomass (Fig. 1a) and also showed highest CFU counts (Fig. 1b). Inquilinus limosus and D. pigrum presented statistically significantly less biomass than $P$. aeruginosa $(P<0.001)$. These species showed similar numbers of CFU (ca. $10^{4} \mathrm{CFU} /$ peg), however $2 \log$ below the cell number produced by $P$. aeruginosa $(P<0.001)$ (Fig. $1 \mathrm{~b})$. Dual-species biofilms formed between $P$. aeruginosa and each of the unconventional species reached cell numbers similar to biofilms of $P$. aeruginosa alone (ca. $10^{6} \mathrm{CFU} /$ peg), however biomass was markedly reduced in mixedspecies biofilms. To confirm that the observed biomass decrease was associated with the amount of extracellular matrix produced by the cells, the protein and polysaccharide content in the matrix was determined using established methods [9]. It was observed that dual-species biofilms of $P$. aeruginosa with I. limosus or $D$. pigrum had a reduction in the matrix of ca. $30 \%$ and $35 \%$, respectively, compared with $P$. aeruginosa biofilms alone (Supplementary Table S1).

Supplementary material related to this article can be found, in the online version, at http://dx.doi.org/10.1016/j.ijantimicag. 2012.04.020.

No statistically significant difference was found between dualspecies biofilms of $P$. aeruginosa and $D$. pigrum growing in TSB and $\mathrm{BHI}$, either in biomass formation (Fig. 1a) or in the number of produced cells (Fig. 1b).

\section{Discussion}

Although it was originally thought that only a limited number of organisms could cause symptomatic infection and lung injury in $\mathrm{CF}$, it has now been shown that the microbial ecology of the CF lung is far more complex than originally thought. In this work, bacteria growing in biofilms were notoriously more difficult to eradicate than when growing planktonically. The wellknown increased resistance associated with biofilms is likely to be multifactorial, depending for instance on the alteration of the metabolism of bacterial cells, on the extra barrier of protection provided by the extracellular matrix that so often encases cells within a biofilm, or even by the spatial arrangement, as well as the number of those bacterial cells that form the layers of the biofilm [10].

Single $P$. aeruginosa biofilms were more resistant to most antibiotics than those formed by emerging species. This may be attributed to the higher biomass and cell numbers obtained for $P$. aeruginosa biofilm formation compared with the other biofilms. The wellknown intrinsic resistance and acquired tolerance to antibiotics of P. aeruginosa biofilms [11] may also support the previous results. Its large and plastic genome favours the species in providing greater adaptability to most hostile environments and to antibiotic treatment, meaning that infection with this species is more arduous to treat [12].

Conversely, emerging bacteria did not show a great ability to form biofilms on the CBD. Nevertheless, the fact that some isolates do not form in vitro biofilms does not impair the ability of these organisms to survive in the patient lung, as recently suggested [13]. Indeed, the reduced capability of I. limosus and D. pigrum to form biofilms was reflected in their sensitivity to most tested antibiotics. These organisms showed significantly less biomass and number of cells than $P$. aeruginosa, making them more vulnerable to antimicrobial agents. The fact that these organisms require an extended incubation time could help explain the slow growth, which consequently results in the lowest amount of biomass and number of produced cells by their single biofilms. In addition, the slimy character of I. limosus bacterial colonies may contribute to the slow growth on the peg surface of the CBD. 

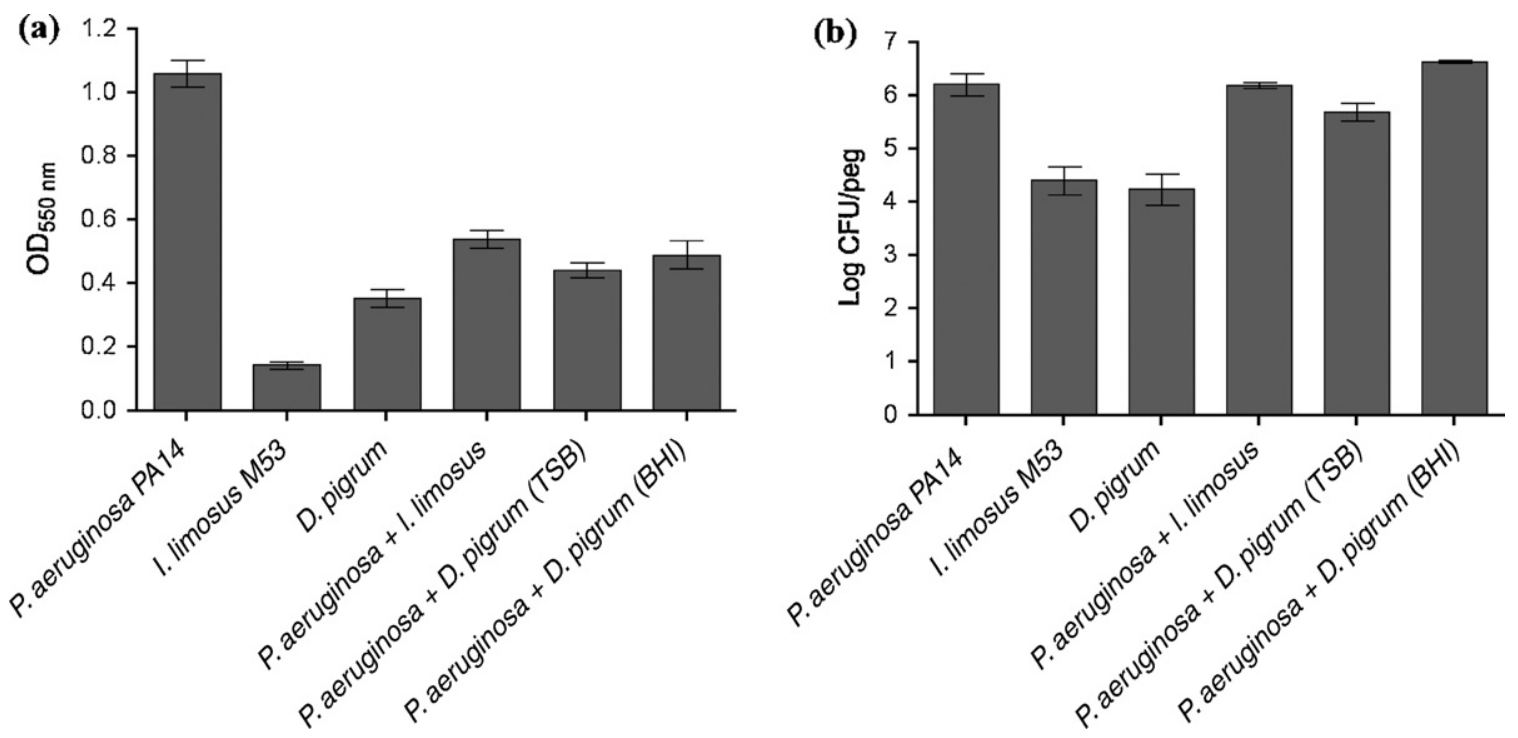

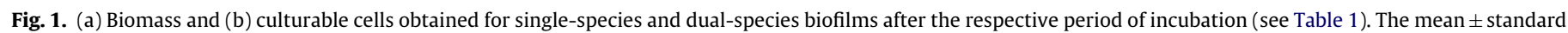
deviation for at least six replicates is illustrated. $\mathrm{OD}_{550}$, optical density at $550 \mathrm{~nm}$; TSB, tryptic soy broth; BHI, brain-heart infusion.

Most studies involving mixed biofilms in CF have only included classical pathogens such as $P$. aeruginosa and B. cepacia [14]. Co-infections of traditional pathogens with rare species in $\mathrm{CF}$ lungs remain largely unexplored, limiting the understanding of the importance of these interspecies interactions. In this study it was demonstrated that most antibiotics presented a poor activity against dual-species biofilms of $P$. aeruginosa with an emerging species. Generally, these biofilms required effective antibiotic concentrations at least equal to that used to kill the same species when in monospecies biofilms. The results obtained by selective media, showing the predominance of $P$. aeruginosa within the consortia, could be the basis for a higher contribution of $P$. aeruginosa to the antibiotic resistances presented by dual-species biofilms. It is recognised that mixed biofilms alter the metabolic activity of the consortium and hence may alter the susceptibility patterns of the population. This can reflect itself for instance in an alteration in the overall biofilm structure and extracellular matrix by both microorganisms, impairing access of antibiotics into the consortium, or by a decreased antibiotic uptake rate through the cell membrane. Here we have shown that independently of the reduced biofilm biomass formed and a decrease in matrix content, the arrangement and even the high number of biofilm-encased cells in mixed-species biofilms was enough to imply an increased resistance on those consortia. This strongly suggests that these organisms and eventually other unusual species might have a great importance in the outcome and treatment of infection in CF. Inaccurate identification of non-conventional pathogens and the disregard for the interactions between all bacteria may lead to ineffective antibiotic therapeutic strategies that could select for antibiotic-resistant pathogens.

In conclusion, although the novel species found in CF appear to be more easily treated by antibiotic therapy than the classical pathogens, they can enhance the antibiotic resistance of mixed populations where they are involved. It is clear that the complex interactions between bacteria in the host play an important role in the complex pathology of the disease and may be often responsible for the increase in antibiotic tolerance. Thus, treatment of infection in CF will probably be more effective in the future by categorising the disease as polymicrobial. It remains to be understood whether in those cases where resistance of $P$. aeruginosa CF biofilms to treatment occurs, we might in fact not be in the presence of a resistant strain of $P$. aeruginosa in the patient but rather of a polymicrobial colonisation.
Note: The data in this manuscript are available in www. biofomics.org, where it is possible to access them for research purposes.

Funding: Financial support was provided by the Portuguese Foundation for Science and Technology [grant SFRH/BD/47613/ 2008 (to SPL) and project PTDC/SAU-ESA/64609/2006].

Competing interests: None declared.

Ethical approval: Not required.

\section{References}

[1] Sibley CD, Parkins MD, Rabin HR, Duan K, Norgaard JC, Surette MG. A polymicrobial perspective of pulmonary infections exposes an enigmatic pathogen in cystic fibrosis patients. Proc Natl Acad Sci USA 2008;105:15070-5.

[2] Ratjen FA. Cystic fibrosis: pathogenesis and future treatment strategies. Respir Care 2009;54:595-605.

[3] Geller DE. Aerosol antibiotics in cystic fibrosis. Respir Care 2009;54:658-70.

[4] Bittar F, Richet H, Dubus JC, Reynaud-Gaubert M, Stremler N, Sarles J, et al. Molecular detection of multiple emerging pathogens in sputa from cystic fibrosis patients. PLoS One 2008;3:e2908.

[5] Sibley CD, Parkins MD, Rabin HR, Surette MG. The relevance of the polymicrobial nature of airway infection in the acute and chronic management of patients with cystic fibrosis. Curr Opin Investig Drugs 2009;10:787-94.

[6] Valenza G, Tappe D, Turnwald D, Frosch M, Konig C, Hebestreit H, et al. Prevalence and antimicrobial susceptibility of microorganisms isolated from sputa of patients with cystic fibrosis. J Cyst Fibros 2008;7:123-7.

[7] Ceri H, Olson ME, Stremick C, Read RR, Morck D, Buret A. The Calgary Biofilm Device: new technology for rapid determination of antibiotic susceptibilities of bacterial biofilms. J Clin Microbiol 1999;37:1771-6.

[8] Harrison JJ, Stremick CA, Turner RJ, Allan ND, Olson ME, Ceri H. Microtiter susceptibility testing of microbes growing on peg lids: a miniaturized biofilm model for high-throughput screening. Nat Protoc 2010;5: $1236-54$

[9] Silva S, Henriques M, Martins A, Oliveira R, Williams D, Azeredo J. Biofilms of non-Candida albicans Candida species: quantification, structure and matrix composition. Med Mycol 2009;47:681-9.

[10] Høiby N, Bjarnsholt T, Givskov M, Molin S, Ciofu O. Antibiotic resistance of bacterial biofilms. Int J Antimicrob Agents 2010;35:322-32.

[11] Tre-Hardy M, Vanderbist F, Traore H, Devleeschouwer MJ. In vitro activity of antibiotic combinations against Pseudomonas aeruginosa biofilm and planktonic cultures. Int J Antimicrob Agents 2008;31: 329-36.

[12] Sykes R. The 2009 Garrod lecture: the evolution of antimicrobial resistance: a Darwinian perspective. J Antimicrob Chemother 2010;65: $1842-52$.

[13] Deligianni E, Pattison S, Berrar D, Ternan NG, Haylock RW, Moore JE, et al. Pseudomonas aeruginosa cystic fibrosis isolates of similar RAPD genotype exhibit diversity in biofilm forming ability in vitro. BMC Microbiol 2010;10: 38.

[14] Tomlin KL, Coll OP, Ceri H. Interspecies biofilms of Pseudomonas aeruginosa and Burkholderia cepacia. Can J Microbiol 2001;47:949-54. 